\title{
Corrosion Monitoring of Steel Structure Coating Degradation
}

\author{
Bojan ZAJEC, Mirjam BAJT LEBAN, Tadeja KOSEC, Vili KUHAR, Andraž LEGAT, Stanislav LENART, Karmen FIFER BIZJAK, \\ Kenneth GAVIN
}

\begin{abstract}
An important aspect regarding the sustainability of steel structures is to ensure the structure is protected from corrosion. A number of surface coatings are available that play an important role in protecting these structures. An important part of the management of these structures is reliable and regular inspection along with methods for early detection of corrosion processes. In this paper, a development and application of sensors for monitoring the steel coating degradation and corrosion damage to steel substrate are presented. An encapsulated corrosion kit with integrated EIS sensors and ER probes was developed. To test its efficiency, steel probes were coated with selected coatings in the laboratory and their performance was assessed under various aggressive atmospheres, including; salt, industrial and humid atmosphere.
\end{abstract}

Keywords: coatings; corrosion; electrochemical impedance spectroscopy; electrical resistance probes; railways; steel structures

\section{INTRODUCTION}

Many of the bridges and viaducts on the Slovenian rail network are more than 150 years old. Whilst early structures were built of stone and masonry, the requirements for longer spans and increased ride quality led to the common use of steel structures [1].

When unprotected, metals corrode while exposed to the atmosphere, with the rate of corrosion depending upon the type of metal, form of construction and physical and chemical composition of atmosphere. In general, corrosion rates increase as the temperature and humidity rise. The most problematic are areas with cyclic wetting and drying periods (night/morning condensation, drying over the day) and areas prone to prolonged condensation, for example structure in shaded areas. In addition, the presence of aggressive ions such are $\mathrm{Cl}^{-}$(commonly found near motorways) and $\mathrm{SO}_{4}{ }^{2-}$ (encountered in industrial areas) also accelerate corrosion processes, especially those of localized nature. The release of iron dust because of wear between rail brake-pads and discs [2, 3] results in a significant danger for corrosion of metal objects situated along the railway tracks. To mitigate these adverse effects the corrosion protection measures are applied, typically through the application of galvanic coatings and/or organic coatings using different types of paints [4]. Such anticorrosion protection does not provide protection over the entire service life, but usually for a period of 15 to 20 years. After that time, protective coatings should be renewed.

The rate of corrosion for protective coatings is in general predictable and well-understood. However, in certain circumstances, for example in crevices, corrosion can often initiate much earlier than is usually expected. Because such locations are difficult to access, even when regular visual inspections are performed, corrosion can be very widespread before it is noticed, leading to, in some cases safety critical conditions, and in others, repairs that could be significantly less costly if the problem had been noticed earlier.

One solution to this problem is to undertake more comprehensive inspections with the increased frequency. The issue here is that trained and skilled inspectors are high-value asset for the infrastructure owner, in addition increased time on the track results in higher risks of incidents involving inspectors and delays to traffic.

Modern railway systems tend to employ various monitoring techniques generally termed as structural health monitoring [5]. Various principles of detection are being used such as acoustics [6], IR thermography [7, 8], eddy currents [9] and various other, mainly MEMStechnology based sensors where the wireless integration provides a complete framework [10-13]. Corrosion hazard represents only one, often underestimated, topic of structural health monitoring [14] and is often implemented in reinforced concrete structures [15-17]. While transducers for many quantities like temperature, strain, acceleration etc. are relatively mature, the transducers for detection and/or measurement of corrosion activity have seen their advancement and application only recently [18]. Out of several methods that provide information on corrosion rate only few can be applied for standalone, field use. So-called corrosion sensors that enable remote monitoring can be broadly divided into two groups depending on the technique they apply [19]. The first group employs various electrochemical methods, while the second group relies on the measurement of other physical quantities that can be related to the corrosion. Since the corrosion is a complex process and appears in various forms, a suitable sensor must be selected for each system, be it corrosion in the pipelines, corrosion of the reinforcement steel, corrosion of rail head surface or some other. In the current study, the long-term corrosion of substrate under the organic coating due to the coating degradation is investigated. Whilst many of the methods are suitable for laboratory use, the reliability of systems in harsh environments like railway networks is less certain.

One of commonly used methods for studying coating degradation is the electrochemical impedance spectroscopy (EIS). EIS is a non-destructive electrochemical technique that can provide a range of information on the condition of coatings [20-22] including; the polarization resistance of the coated metal, the capacitive behaviour of organic coatings, water uptake into the coating etc. In a corrosive media, this method can provide information on polarization resistance, $R_{\mathrm{p}}$ of a certain material in a particular environment (steel in concrete, stainless steel in a strong acid, TiAlV alloy in simulated body fluid etc.). The impedance spectrum at the 
potential of the open circuit potential or at potential of choice is collected by measuring impedances at several different frequencies. The impedance data can be presented in different forms, with Nyquist and Bode plot being the most commonly used form of graphical representation. The impedance data can be interpreted based on equivalent electrical circuits using fitting programs. Equivalent circuits represent the physical properties of the metal and its coating in the system. Different properties can be deduced from the EIS results such as the effective thickness of the film and the total overall polarization resistances $\left(R_{\mathrm{p}}\right)$ after different immersion times thus showing the extent of corrosion degradation of the coating and steel substrate. EIS offers broad information on the coating quality, however it requires two additional electrodes and dedicated electronics and is therefore mostly limited to the laboratory use. Several attempts have been to adapt it for the field use [23, 24] and also to decrease the cost of the electronics [25].

Electrical resistance (ER) probes were originally developed for monitoring corrosion of reinforcement steel in concrete structures. It was shown [26] that this is a reliable method for measuring the cumulative corrosion damage of steel in concrete. It enabled a good assessment of the average general corrosion rate that occurred over defined time intervals. However, the response of these probes to localized corrosion types and transient events was limited. ER probes have been applied also for measurement of atmospheric corrosion [27] and corrosion in neutral solutions while examining the protection by corrosion inhibitor or cathodic protection [28]. According to our literature survey this study is the first one investigating the application of ER probes for assessment of corrosion under the coating and correspondingly its degradation. ER probes are based on physical response to corrosion, and therefore they are not directly susceptible to variations of electrochemical parameters and changes in the electrolyte. The main advantage of this method in comparison to the conventional electrochemical sensor approach is that it does not need an electrolyte present for operation. However, this method cannot detect early stage of coating degradation, but only initiation and propagation of corrosion on the substrate.

Within the European Commission FP7 SMARTRAIL project [29] a corrosion kit that combines EIS and ER sensors for monitoring of corrosion degradation of coating was developed. The design of the kit, its operation and also results of measurements in the laboratory are presented in this paper.

\section{EXPERIMENT (DESCRIPTION OF CORROSION KIT)}

The corrosion kit was specially designed for the measurement of the corrosion properties of coatings and corrosion on the steel substrate covered by a protective coating. This corrosion kit, Fig. 1, combines two different measurement systems; electrochemical impedance spectroscopy (EIS) and electrical resistance probes (ER). For each technique a special sensor is required with both methods enabling non-destructive measurement of different corrosion properties and processes. For the validation phase, three different types of coating were used. A primary coating (with a thickness of $80 \mu \mathrm{m}$ ), a primary plus intermediate coating (220 $\mu \mathrm{m}$ total thickness) and primary, intermediate and top coating together in one system $(280 \mu \mathrm{m}$ total thickness) (Figs. 1 and 2).

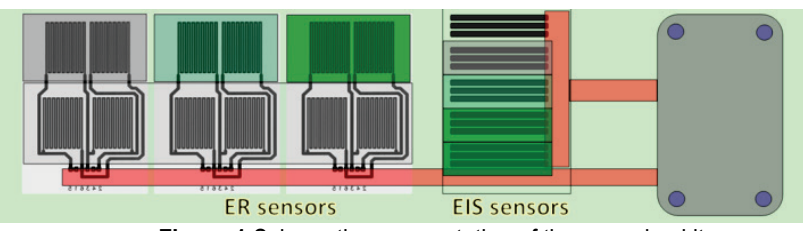

Figure 1 Schematic representation of the corrosion kit

The primer is a two-part high-build epoxy paint containing zinc phosphate, its commercial name is Epolor Special B. The intermediate coating is a two-part, highbuild, high solids, modified epoxy paint with low level of solvents. It exhibits good penetration properties and is commercially available as Epolor HB. The top coating was a two-part, high-build polyurethane paint with the commercial name Bukolit Emajl HB B. All three paints are produced by Helios, Slovenia. The paints used for the primer and intermediate layers have good mechanical, thermal and water (incl. salt water) resistance and also good resistance against caustics and organic solvents. They have medium resistance against acids and poor resistance against UV light. The applied top coating displays excellence in all the above mentioned properties, with the exception of its resistance against acids which is classified as medium. The selection of coatings is in agreement with the compatibility list provided by the producer in data sheets.

Three identical kits were prepared and exposed to the three artificial atmospheres - one in a salt spray chamber (exposure according to the standard EN ISO 9227), one to a humid chamber (EN ISO 6270) and one was placed into an industrial $-\mathrm{SO}_{2}$ chamber (EN ISO 3231).

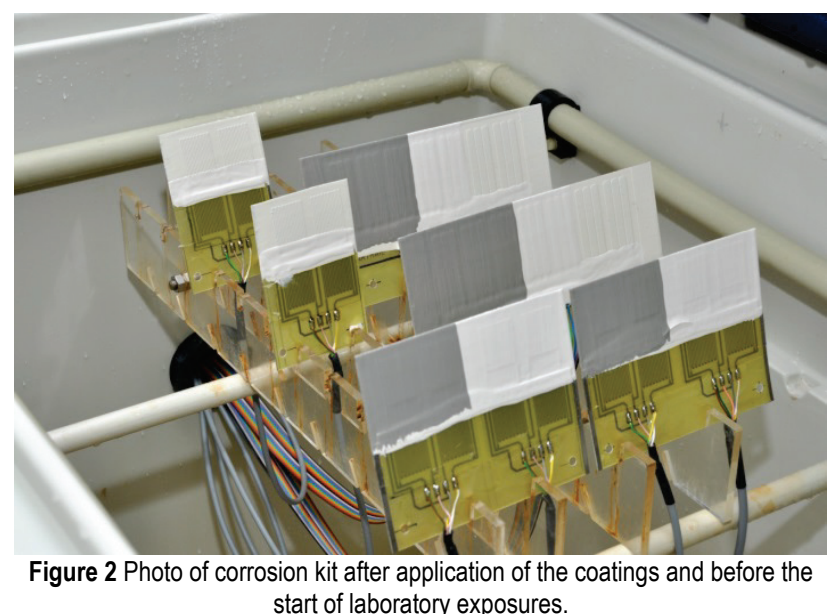

In the laboratory the measurements of the EIS and ER probes, contained in the corrosion kit, were carried out periodically for up to 283 days in total.

\subsection{Electrochemical Impedance (EIS)}

In order to obtain measurements using this method it was necessary to temporarily remove the corrosion kit from the chamber where it was exposed. Before each measurement, the corrosion sensors (working electrode) were immersed for 1 hour into the testing solution of $0.1 \mathrm{M}$ 
$\mathrm{NaCl}$ (in the case of kits exposed into salt spray chamber) or $0.1 \mathrm{M} \mathrm{NaHCO}$ s solution (kits exposed into humid or industrial chamber) to achieve steady state conditions in the electrolyte. Impedance spectra were obtained within the frequency range $100 \mathrm{kHz}-10 \mathrm{mHz}$ while the amplitude of the excitation signal was $20 \mathrm{mV}$ RMS. All measurements were performed by using calomel electrode $(E=0.245 \mathrm{~V}$ vs. standard hydrogen electrode) as a reference.

\subsection{Electrical Resistance Probes $(E R)$}

Electrical resistance $(E R)$ probes were constructed from 4 resistors made with the printed circuit board etching technique (PCB) using a $240 \mu \mathrm{m}$ thick carbon steel plate. The width of the long electrical leads serving as the resistors is about $0.5 \mathrm{~mm}$. The nominal steel surface area of one sensor element is $3.85 \mathrm{~cm}^{2}$. These resistors were placed as a Wheatstone bridge, Fig. 3. Two of the resistors formed the reference and protected against corrosion with another semi-transparent glass-fibre resin plate and glued with the transparent epoxy resin. The other two were unprotected and coated only with the coating to be investigated. Since these two resistor elements were exposed to corrosion, the nominal surface area of the corroding section was $7.7 \mathrm{~cm}^{2}$.
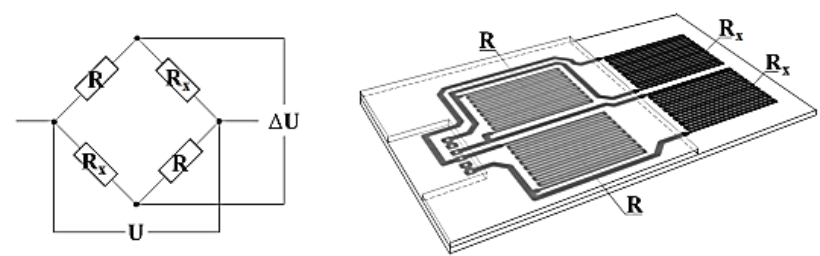

Figure 3 Scheme of Wheatstone bridge in an electrical resistance $(E R)$ sensor. $\mathrm{R}$ denotes reference resistors, while $\mathrm{R}_{x}$ denotes resistors that change due to the corrosion process.

\section{MONITORING RESULTS AND DISCUSSION 3.1 Results of Visual Examinations during and after Exposures in Environmental Chambers}

The most aggressive atmosphere to which the measurement kits were exposed was the industrial $\left(\mathrm{SO}_{2}\right)$ atmosphere, followed by the salt spray and the humid atmosphere. The measurement kits were exposed in the chambers for 289 days (6936 hours) in total. The results of visual examination revealing the first signs of corrosion on different coatings and in different atmospheres (chambers) are presented in Tab. 1.

From Tab. 1, it can be observed that for the salt spray chamber, corrosion was detected for the primary coating and primary + intermediate coating and no corrosion was measured for the primary, intermediate and top coating case. For the industrial atmosphere, only the primary coating developed corrosion during the experiment. For the humid atmosphere, none of the coatings developed visible corrosion during the test period.

Table 1 Exposure time when the first signs of corrosion under coating were visually observed

\begin{tabular}{|c|c|c|c|}
\hline $\begin{array}{c}\text { Coating system / } \\
\text { Atmosphere }\end{array}$ & $\begin{array}{c}\text { Salt spray } \\
\text { chamber }\end{array}$ & $\begin{array}{c}\text { Humid } \\
\text { chamber }\end{array}$ & $\begin{array}{c}\text { Industrial }\left(\mathrm{SO}_{2}\right) \\
\text { chamber }\end{array}$ \\
\hline Primary coating & after $27 \mathrm{~h}$ & $\begin{array}{c}\text { no corrosion } \\
\text { detected }\end{array}$ & $\begin{array}{c}\text { after } 25 \text { cycles }= \\
600 \mathrm{~h}\end{array}$ \\
\hline $\begin{array}{c}\text { Primary }+ \\
\text { intermediate coating }\end{array}$ & after $2136 \mathrm{~h}$ & $\begin{array}{c}\text { no corrosion } \\
\text { detected }\end{array}$ & $\begin{array}{c}\text { no corrosion } \\
\text { detected }\end{array}$ \\
\hline $\begin{array}{c}\text { Primary }+ \\
\text { intermediate }+ \\
\text { top coating }\end{array}$ & $\begin{array}{c}\text { no corrosion } \\
\text { detected }\end{array}$ & $\begin{array}{c}\text { no corrosion } \\
\text { detected }\end{array}$ & $\begin{array}{c}\text { no corrosion } \\
\text { detected }\end{array}$ \\
\hline
\end{tabular}

Table 2 The development of corrosion versus time on samples with primary coatings in the salt spray chamber

\begin{tabular}{|c|c|c|c|c|}
\hline $27 \mathrm{~h}$ & & $456 \mathrm{~h}$ & $2856 \mathrm{~h}$ \\
\hline
\end{tabular}

Table 3 The development of corrosion versus time on samples exposed in the industrial chambe

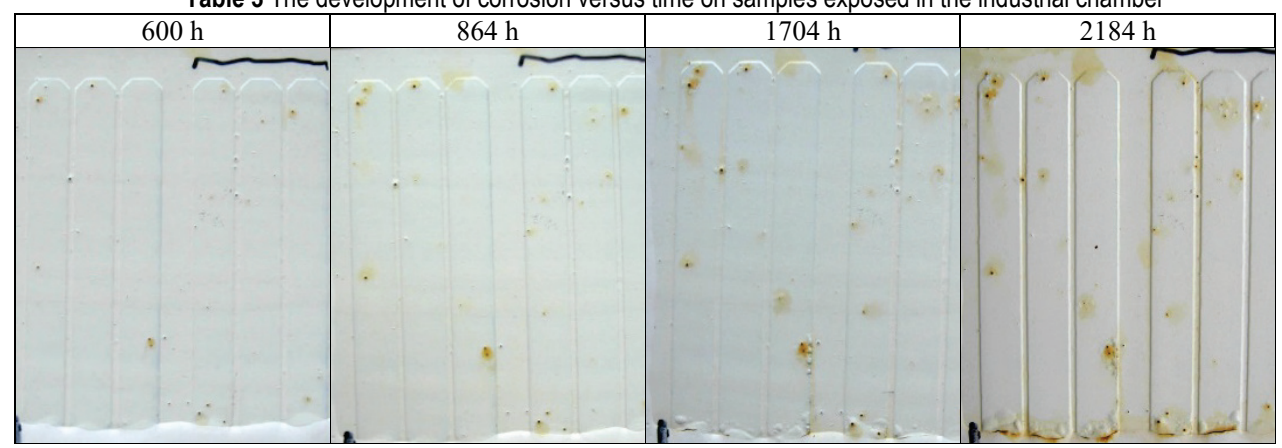


Deterioration of the primary coating was evident in the two harshest environmental chambers (i.e. the salt spray and industrial chamber) and more evident on EIS sensors than on ER sensors. Namely, EIS sensors differ significantly from ER sensors in construction, and consequently also on adhesion of organic coating. In our opinion, this is the most important reason for different corrosion appearance under the coating.

In Tabs. 2 and 3 images of the development of corrosion with time of exposure for the primary coating on samples exposed in the salt spray and industrial chamber are shown.

In Tab. 4 photos of the development of corrosion with time on samples with primary and intermediate coatings exposed in the salt spray chamber are shown. No corrosion

was observed on any of exposed coated sensors in humid chamber.

Table 4 Corrosion development with time for samples with primary and intermediate coatings exposed in the salt spray chamber
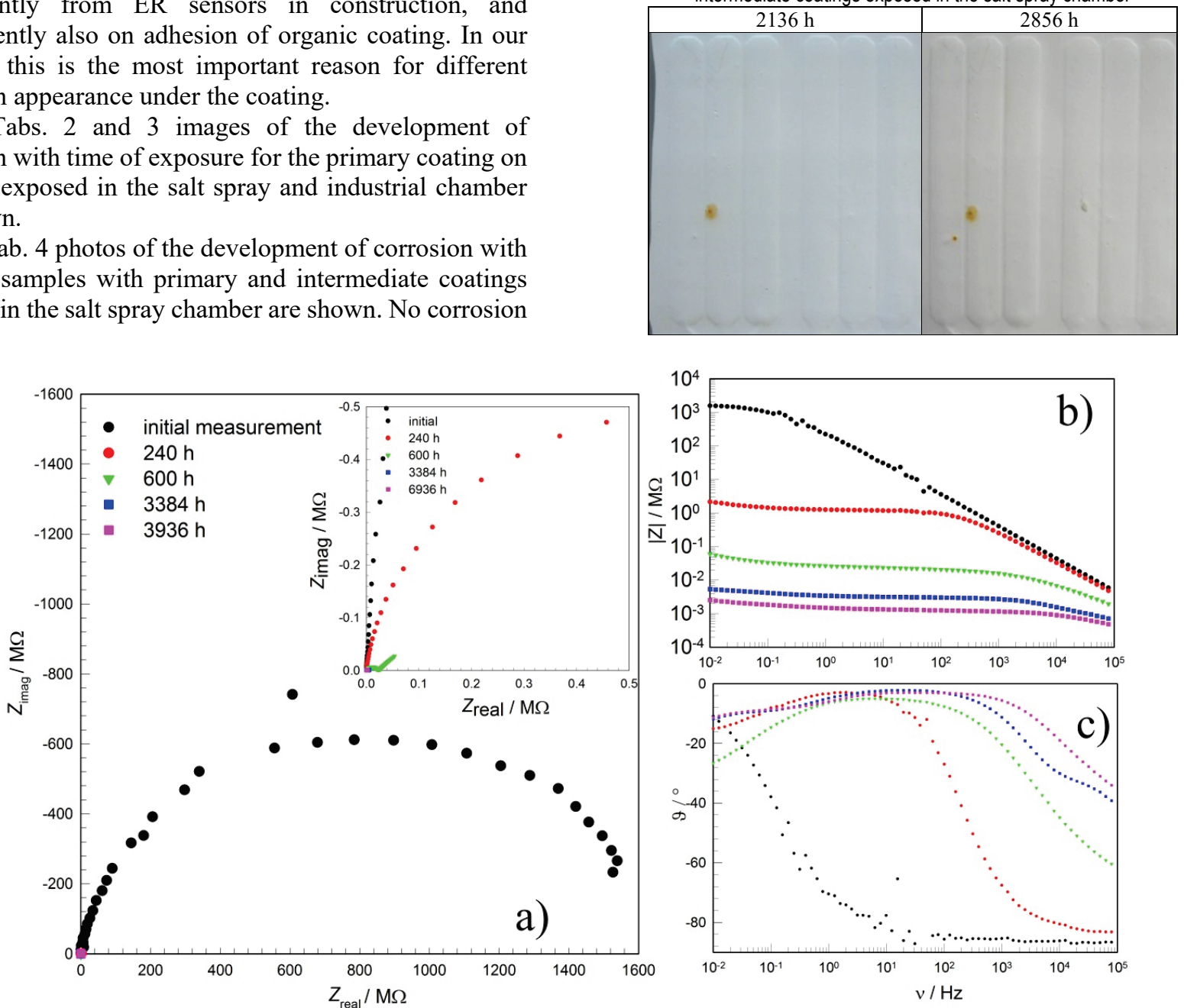

Figure 4 Results of EIS measurement of primary coating in salt spray chamber at different exposure times

\subsection{Results of EIS Measurement}

The EIS sensors enable the user to monitor changes of the coating properties as the change of the impedance response versus time. Reductions in impedance are indicative of degradation in the coating. In addition, the difference in performance of the different protective systems can be compared by absolute impedance values obtained under the same conditions.

In Figure 4, the impedance responses for primary coatings tested in the salt spray chamber are presented. The measurements enable the condition of the coating to be tracked versus time. The impedance is plotted on the Nyquist diagrams (Fig. 4a) and on Bode plots (Fig. 4b and $4 \mathrm{c}$ ). From both plots it can be observed that the primary coating decayed with time, as observed by lowering impedance response in Nyquist diagram and by lowering the impedance magnitude at lower measured frequency in Bode plots, similar observations can be found in Tab. 5 where the values of polarization resistances, gained from the fitting of the spectra, are presented. Also, in Tab. 5, the slow decay of intermediate and top coating is observed.
Similar results of measurements were observed during the exposure of sensors in industrial chamber.

Fig. 5 shows the state of the coatings after exposure in salt spray chamber for nine months. The impedance response is the lowest for the primary coating and the highest for the system primary + intermediate coating.

The polarization resistance values, $R_{\mathrm{p}}$ after nine months of exposure in the salt spray chamber were: $0.00262 \mathrm{M} \Omega / \mathrm{cm}^{2}$ for primary, $5.734 \mathrm{M} \Omega / \mathrm{cm}^{2}$ for intermediate and $1.74 \mathrm{M} \Omega / \mathrm{cm}^{2}$ for the top coating. The values of polarization resistance show the corrosion resistivity properties. The lower the value is, the higher the corrosion experienced [30]. Impedance results for sensors with different protections in the salt spray chamber after exposure for nine months show that basic protection with primary coating failed, whereas the intermediate and top coatings show relatively good anticorrosion properties as observed from the impedance response in Nyquist diagram (Fig. 5a) and from total impedance at lowest measured frequency in the Bode diagrams (Fig. 5b and 5c). 

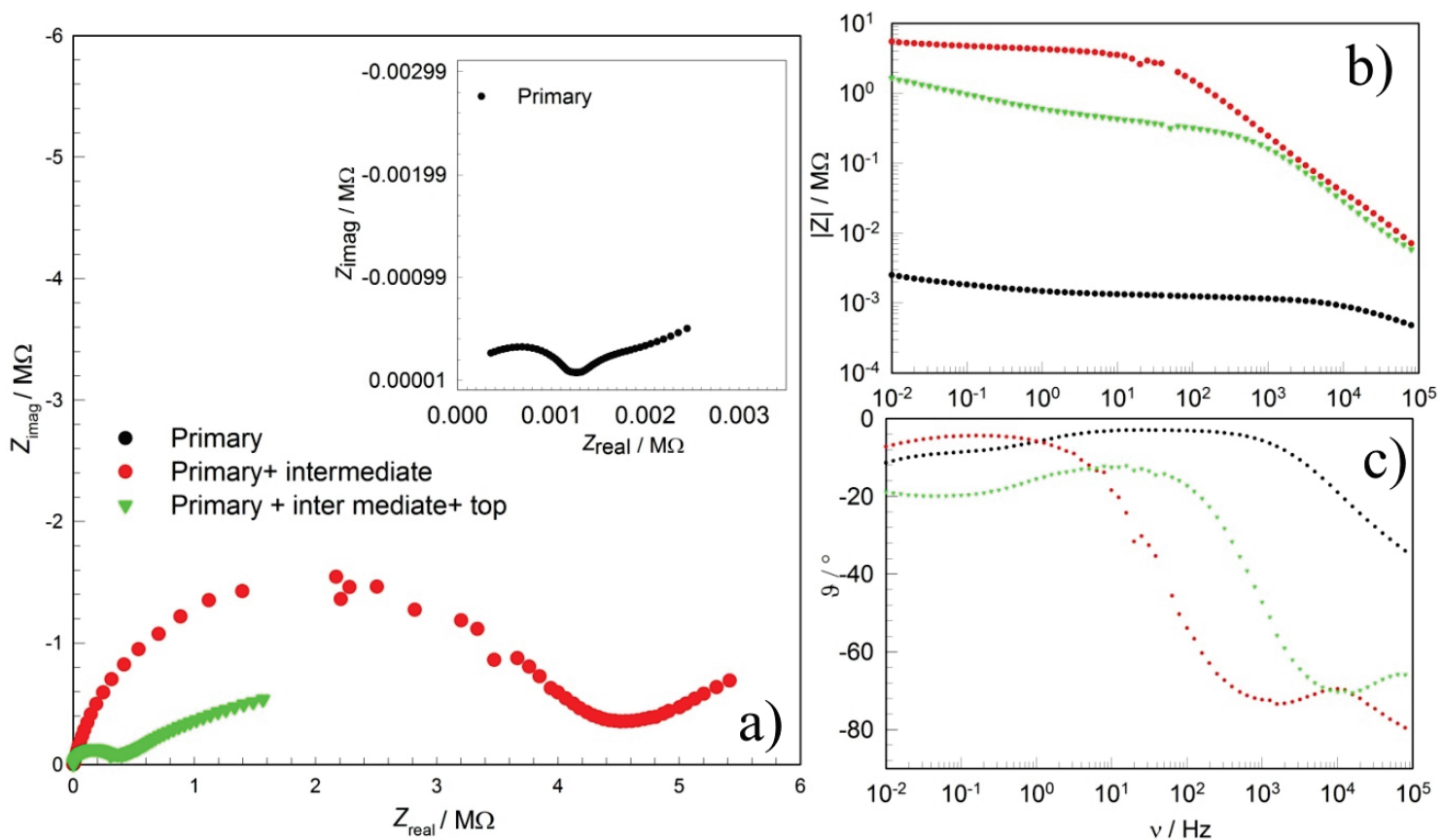

Figure 5 Results of EIS measurements in salt spray chamber after 289 days (6936 h) of exposure (approx. 9 months)

Impedance measurements thus allow the "corrosion state" of the coatings which in general and as expected slowly decay with time, to be monitored. The slowest decay rate is observed for the intermediate coating in the salt spray chamber and the most rapid decay is observed for the primary coating (Tab. 5). The reason that the top coating performed somewhat inferior in comparison to the intermediate coating could be due to minor problems in quality controlled related to the application of the top coating.

Table 5 Polarization resistance values for different coatings from salt spray chamber at different exposure times

\begin{tabular}{|c|c|c|c|c|c|}
\hline $\mathrm{M} \Omega \mathrm{cm}^{2}$ & Initial & $240 \mathrm{~h}$ & $600 \mathrm{~h}$ & $3384 \mathrm{~h}$ & $6936 \mathrm{~h}$ \\
\hline Primary coating & 1620 & 2.23 & 0.063 & 0.0056 & 0.0026 \\
\hline Intermediate & 3280 & 75.1 & 34.4 & 13.5 & 5.73 \\
\hline Top coating & 31510 & 26.9 & 19.5 & 5.56 & 1.74 \\
\hline
\end{tabular}

Scully [30] demonstrated that, in the case of lowest observed frequency, absolute impedance can be correlated to the polarization resistance, $R_{\mathrm{p}}$, of the observed alloy in a conductive solution. Fig. 6 presents corrosion rate $C R$, in $\mu \mathrm{m} /$ year, calculated by using Faraday's law [31] by the following formula:

$$
C R=3.27 \frac{j_{\text {corr }}}{d} \frac{W}{n},
$$

where $j_{\text {corr }}$ is the corrosion current density in $\mu \mathrm{A} / \mathrm{cm}^{2}, d$ the density in $\mathrm{g} / \mathrm{cm}^{3}, W$ the atomic weight (dimensionless) and $n$ the number of electrons required to oxidize an atom. For steel, the density and atomic weights are $d=7.87 \mathrm{~g} / \mathrm{cm}^{3}$ and 55.8, respectively and $n=2$.

The corrosion current density, $j_{\text {corr }}$, in $\mathrm{A} \mathrm{cm}^{-2}$ was calculated from the Stern-Geary equation [32]

$j_{\text {corr }}=\frac{B}{R_{\mathrm{p}} \cdot A}$, where $B$ is the Stern-Geary coefficient in $\mathrm{V}, R_{\mathrm{p}}$ polarization resistance in $\Omega$ and $A$ is surface of exposed metal in $\mathrm{cm}^{2}$ $\left(2.75 \mathrm{~cm}^{2}\right)$. Corrosion current density is calculated using the $R_{\mathrm{p}}$ values first (in $\Omega$ ) and a $B$-value of $26 \mathrm{mV}$ [19].

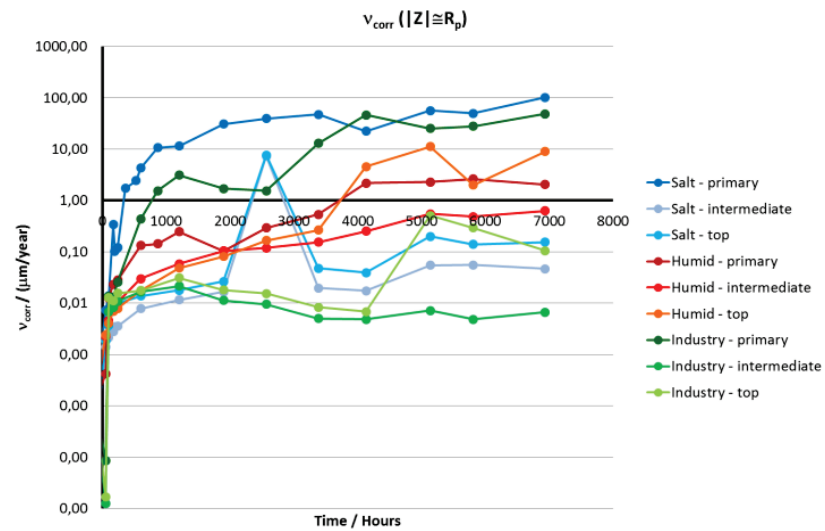

Figure 6 Corrosion rates (in logarithmic scale) obtained from EIS measurements on sensors covered by three types of coatings in salt spray (blue lines), humid (red lines) and industrial chamber (green lines)

From the results, presented in Fig. 6, it can be observed that corrosion rate increased largely on primary coatings. It is the greatest for the primary coating, exposed to salt and industrial chamber, but smaller for the primary coating exposed to humidity chamber.

Intermediate coatings show the highest corrosion rate in humidity chamber and smaller corrosion rate in salt and industrial chambers. Similar observations were found for the top coatings. The overall corrosion rate is smaller than for primary coating, but the corrosion rate is the highest in humidity chamber, following with salt spray and industrial chamber.

\subsection{Results of Measurement on ER probes}

Coatings of ER probes were not degraded so severely as coating of EIS sensors. Reasons for that are described in 3.1. Consequently, corrosion of steel substrate of ER 
probes was not so pronounced as it was the case with steel substrate of EIS sensors. Thickness reductions of ER sensors are presented in Fig. 7, and the surface images of the most destroyed coatings of ER sensors in Tab. 6. Early failures of ER sensors, covered by primary and primary + intermediate coating which were exposed to industrial chamber with addition of aggressive $\mathrm{SO}_{4}{ }^{2-}$, are a consequence of corrosion between glass-fiber resin plate and steel substrate of reference resistor of ER sensor (see Fig. 8).

However, from Fig. 7 the reduction of thickness of sensor covered by primary coating and the one exposed to salt spray chamber can be clearly seen. The reduction of the thickness is approximately $10 \mu \mathrm{m}$ in 9 months. Change of the thickness of other sensors was negligible.

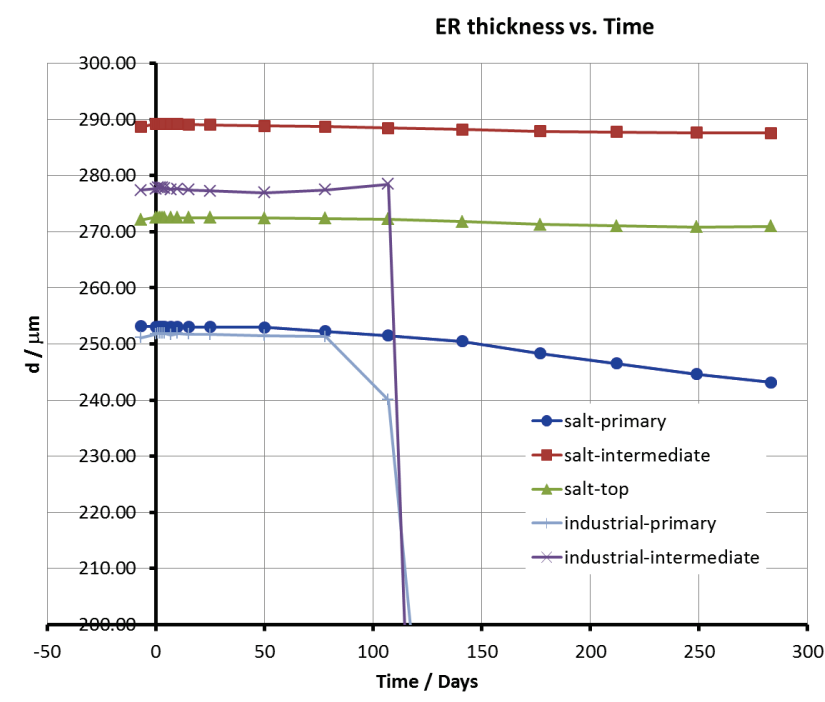

Figure 7 Measurements of ER sensors thickness reduction in different environmental chambers

Table 6 Surface condition of the most destroyed coatings of ER sensors exposed to salt spray chamber for 456,1056 and 2856 hours

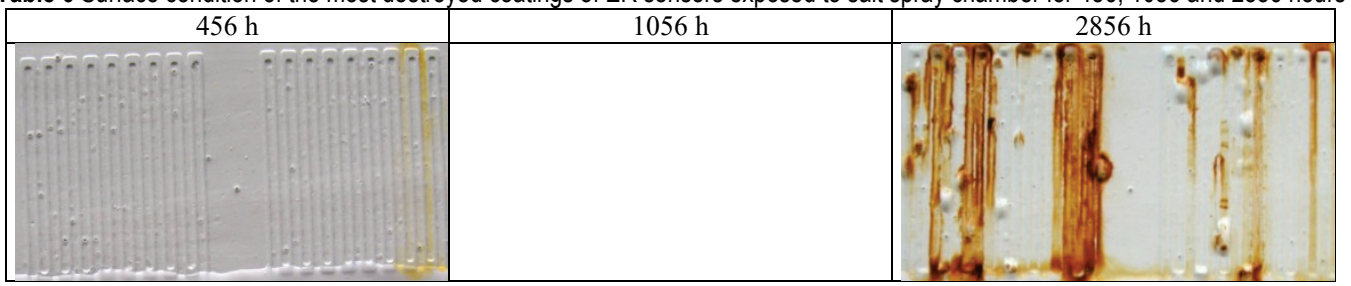

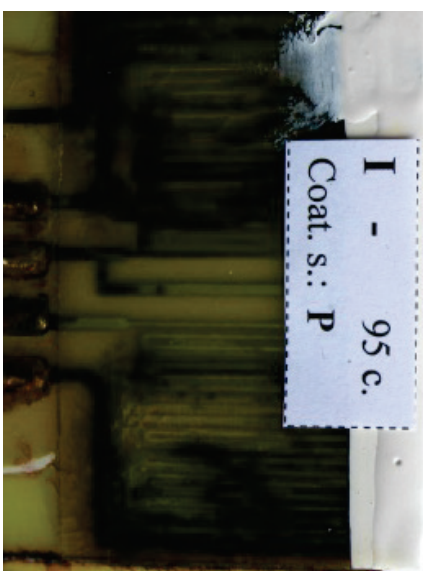

Figure 8 Corrosion between laminate (yellow) and steel substrate of ER senso exposed to the industrial chamber (after 2280 hours) - left part of the sensor

Corrosion of steel under the coating is a slow process, since the protective coating must first collapse; only then the corrosion of steel starts. Nevertheless, ER probes provide relevant and accurate method for detection of the initiation as well as propagation of corrosion under coating on steel surfaces. The use of this method is particularly well suited to inaccessible places and locations which are the weakest from the point of view of corrosion (for example dead angles, crevices, etc.).

\section{CONCLUSION}

Measurement kit with sensors for monitoring of electrochemical impedance and corrosion rate by ER probes was constructed in order to enable on-site monitoring of corrosion degradation of steel under coatings. Several exposures simulating different real environments were carried out in laboratory to study reliability of such monitoring. On the other hand, these studies were used also to recognize disadvantages of such system.

From the laboratory exposure the following conclusions can be drawn:

- EIS as a monitoring technique is efficient tool to monitor protection ability of the system under investigation.

- However, conventional measurement of EIS on constructed corrosion kit is not possible without using of electrolyte and conventional reference electrode.

- At the moment, EIS for this corrosion kit can be measured only in a configuration as in the laboratory.

- However, it was shown, that monitoring of corrosion rate by ER probes is a very reliable and sensitive method which enables early detection of corrosion initiation.

- In addition, ER method is very suitable for monitoring of corrosion processes in real environment. Sensors can be equipped by data-loggers to aggregate data remotely.

In the case of work within SmartRail project, unexpected failure of sensors exposed to aggressive industrial environment was observed where failure of reference part of sensor occurred due to technology of protection of this reference part of sensors. The team is looking forward to find appropriate solution to avoid this type of failures (better materials for sensors production, design improvement) in the future and also searching for solution to simplify EIS measurements on real objects. 


\section{Acknowledgement}

Project was founded by the EU 7th Framework Programme under call SST.2011.5.2-6 Cost-effective improvement of rail transport infrastructure. Grant agreement no: 285683 .

\section{REFERENCES}

[1] Bogić, M. (2012). Bridges of Slovenia. Archit. Bull., 195196, 43-53.

[2] Akkus, A. \& Yeğin, M. (2014). Research on wear rate and mechanical properties of brake sabots (shoes) used in railway rolling stocks. Int. J. Appl. Sci. Technol., 4(7), 7684.

[3] Burkhardt, M. et al. (2008). Diffuse release of environmental hazards by railways. Desalination, 226(1), 106-113. https://doi.org/10.1016/j.desal.2007.02.102

[4] Schweitzer, P. A. (2006). Corrosion of Linings \& Coatings: Cathodic and Inhibitor Protection and Corrosion Monitoring. CRC Press. https://doi.org/10.1201/9780849382482

[5] Barke, D. \& Chiu, W. K. (2005). Structural Health Monitoring in the Railway Industry: A Review. Struct. Health Monit., 4(1), 81-93. https://doi.org/10.1177/1475921705049764

[6] Gong, Z. et al. (1992). Acoustic emission monitoring of steel railroad bridges. Mater. Eval., 50(7), 883-887. https://doi.org/10.1016/0963-8695(92)90655-Z

[7] Bagavathiappan, S. et al. (2013). Infrared thermography for condition monitoring - A review. Infrared Phys. Technol., 60, 35-55. https://doi.org/10.1016/j.infrared.2013.03.006

[8] Netzelmann, U. et al. (2016). NDT of railway components using induction thermography. AIP Conf. Proc., 1706(1), 150001. https://doi.org/10.1063/1.4940613

[9] Sodano, H. A. (2007). Development of an Automated Eddy Current Structural Health Monitoring Technique with an Extended Sensing Region for Corrosion Detection. Struct. Health Monit., 6(2), 111-119. https://doi.org/10.1177/1475921706072065

[10] Flammini, F. et al. (2010). Towards Wireless Sensor Networks for railway infrastructure monitoring. In: Proceedings of the International Conference on Electrical Systems for Aircraft, Railway and Ship Propulsion, ESARS 2010 / Bologna, 1-6. https://doi.org/10.1109/ESARS.2010.5665249

[11] Hodge, V. J. et al. (2015). Wireless Sensor Networks for Condition Monitoring in the Railway Industry: A Survey. IEEE Trans. Intell. Transp. Syst., 16(3), 1088-1106. https://doi.org/10.1109/TITS.2014.2366512

[12] Qiao, G. et al. (2011). Remote corrosion monitoring of the RC structures using the electrochemical wireless energyharvesting sensors and networks. NDT E Int., 44(7), 583588. https://doi.org/10.1016/j.ndteint.2011.06.007

[13] Shafiullah, G. M. et al. (2007). Survey of Wireless Communications Applications in the Railway Industry. In: The $2^{\text {nd }}$ International Conference on Wireless Broadband and Ultra Wideband Communications (AusWireless 2007) / Sydney, 65-65. https://doi.org/10.1109/AUSWIRELESS.2007.74

[14] Xu, Y. L. \& Xia, Y. (2011). Structural Health Monitoring of Long-Span Suspension Bridges. CRC Press. https://doi.org/10.1201/b13182

[15] Broomfield, J. P. et al. (2002). The use of permanent corrosion monitoring in new and existing reinforced concrete structures. Cem. Concr. Compos., 24(1), 27-34. https://doi.org/10.1016/S0958-9465(01)00024-5

[16] Fuhr, P. L. \& Huston, D. R. (1998). Corrosion detection in reinforced concrete roadways and bridges via embedded fiber optic sensors. Smart Mater. Struct., 7(2), 217. https://doi.org/10.1088/0964-1726/7/2/009
[17] Raupach, M. et al. (2014). Corrosion of Reinforcement in Concrete: Monitoring, Prevention and Rehabilitation Techniques. Woodhead Publishing.

[18] Groysman, A. (2009). Corrosion monitoring. Corros. Rev. 27(4-5), 205-343. https://doi.org/10.1515/CORRREV.2009.27.4-5.205

[19] Yang, L. (2008). Techniques for corrosion monitoring. Elsevier.

[20] Bonora, P. L. et al. (1996). Electrochemical impedance spectroscopy as a tool for investigating underpaint corrosion. Electrochimica Acta, 41(7), 1073-1082. https://doi.org/10.1016/0013-4686(95)00440-8

[21] Kendig, M. \& Scully, J. (1990). Basic Aspects of Electrochemical Impedance Application for the Life Prediction of Organic Coatings on Metals. Corrosion, 46(1), 22-29. https://doi.org/10.5006/1.3585061

[22] Mansfeld, F. (1995). Use of electrochemical impedance spectroscopy for the study of corrosion protection by polymer coatings. J. Appl. Electrochem., 25(3), 187-202. https://doi.org/10.1007/BF00262955

[23] Davis, G. D. et al. (2002). Coating evaluation and validation of accelerated test conditions using an in-situ corrosion sensor. J. Coat. Technol., 74(935), 69-74. https://doi.org/10.1007/BF02697959

[24] Pejcic, B. \& De Marco, R. (2006). Impedance spectroscopy: Over 35 years of electrochemical sensor optimization. Electrochimica Acta, 51(28), 6217-6229. https://doi.org/10.1016/j.electacta.2006.04.025

[25] Angelini, E. et al. (2014). An Arduino-based EIS with a logarithmic amplifier for corrosion monitoring. In: Proceedings 2014 IEEE International Instrumentation and Measurement Technology Conference (I2MTC) / Montevideo, 905-910. https://doi.org/10.1109//2MTC.2014.6860873

[26] Legat, A. (2007). Monitoring of steel corrosion in concrete by electrode arrays and electrical resistance probes. Electrochimica Acta, 52(27), 7590-7598. https://doi.org/10.1016/j.electacta.2007.06.060

[27] Cai, J.-P. \& Lyon, S. B. (2005). A mechanistic study of initial atmospheric corrosion kinetics using electrical resistance sensors. Corros. Sci., 47(12), 2956-2973. https://doi.org/10.1016/j.corsci.2005.04.011

[28] Li, S. et al. (2007). Application of steel thin film electrical resistance sensor for in situ corrosion monitoring. Sens. Actuators B Chem., 120(2), 368-377. https://doi.org/10.1016/j.snb.2006.02.029

[29] Smartrail project homepage: http://smartrail.fehrl.org/

[30] Scully, J. R. (2000). Polarization resistance method for determination of instantaneous corrosion rates. Corrosion, 56(2), 199-218. https://doi.org/10.5006/1.3280536

[31] ASTM Designation: G 102 - 89 (Reapproved 1999), Standard Practice for Calculation of Corrosion Rates and Related Information form Electrochemical Measurements. 1999.

[32] Stern, M. \& Geary, A. L. (1957). Electrochemical Polarization I. A Theoretical Analysis of the Shape of Polarization Curves. J. Electrochem. Soc., 104(1), 56-63. https://doi.org/10.1149/1.2428496

\section{Contact information}

\section{Bojan ZAJEC}

Slovenian National Building and Civil Engineering Institute

Dimičeva 12, 1000 Ljubljana, Slovenia

E-mail: bojan.zajec@zag.si

\section{Mirjam BAJT LEBAN}

Slovenian National Building and Civil Engineering Institute

Dimičeva 12, 1000 Ljubljana, Slovenia 
Current affiliation:

Akrapovič d.d.

Malo Hudo 8a, 1295 Ivančna Gorica, Slovenia

Tadeja KOSEC

Slovenian National Building and Civil Engineering Institute

Dimičeva 12, 1000 Ljubljana, Slovenia

\section{Vili KUHAR}

Slovenian National Building and Civil Engineering Institute Dimičeva 12, 1000 Ljubljana, Slovenia

\section{Andraž LEGAT}

Slovenian National Building and Civil Engineering Institute

Dimičeva 12, 1000 Ljubljana, Slovenia

\section{Stanislav LENART}

Slovenian National Building and Civil Engineering Institute Dimičeva 12, 1000 Ljubljana, Slovenia

\section{Karmen FIFER BIZJAK}

Slovenian National Building and Civil Engineering Institute

Dimičeva 12, 1000 Ljubljana, Slovenia

\section{Kenneth GAVIN}

Faculty of Civil Engineering and Geoscience, TU Delft Building 23, Stevinweg 1, 2628 CN Delft, Netherlands 